\title{
Exocrine pancreatic disorders in transsgenic mice expressing human keratin 8
}

\author{
M. Llanos Casanova, ${ }^{1}$ Ana Bravo, ${ }^{2}$ Angel Ramírez, ${ }^{1}$ Gabriela Morreale de Escobar, ${ }^{3}$ \\ Felipe Were, ${ }^{4}$ Glenn Merlino, ${ }^{5}$ Miguel Vidal, ${ }^{4}$ and José L. Jorcano ${ }^{1}$
}

${ }^{1}$ Cell and Molecular Biology, Centro de Investigaciones Energéticas, Medioambíentales y Technológicas, E-28040 Madrid, Spain
2Department of Animal Pathology, Veterinary School, University of Santiago de Compostela, E-27002 Lugo, Spain
${ }^{3}$ Unidad de Endocrinología Molecular, Instituto de Investigaciones Biomédicas, E-28029 Madrid, Spain
${ }^{4}$ Department of Developmental and Cell Biology, Centro de Investigaciones Biológicas, E-28006 Madrid, Spain
${ }_{5}^{5}$ Laboratory of Molecular Biology, National Cancer Institute, National Institutes of Health, Bethesda, Maryland 20892, USA

Address correspondence to: José L. Jorcano, Cell and Molecular Biology, Centro de Investigaciones Energéticas,

Medioambíentales y Technológicas, Av. Complutense 22, E-28040 Madrid, Spain. Phone: 34-91-346-65-98; Fax: 34-91-346-63-93;

E-mail: jorcano @ciemat.es

Received for publication September 25, 1998, and accepted in revised form May 4, 1999.

\begin{abstract}
Keratins K8 and K18 are the major components of the intermediate-filament cytoskeleton of simple epithelia. Increased levels of these keratins have been correlated with various tumor cell characteristics, including progression to malignancy, invasive behavior, and drug sensitivity, although a role for K8/K18 in tumorigenesis has not yet been demonstrated. To examine the function of these keratins, we generated mice expressing the human $\mathrm{K} 8(b k 8)$ gene, which leads to a moderate keratin-content increase in their simple epithelia. These mice displayed progressive exocrine pancreas alterations, including dysplasia and loss of acinar architecture, redifferentiation of acinar to ductal cells, inflammation, fibrosis, and substitution of exocrine by adipose tissue, as well as increased cell proliferation and apoptosis. Histological changes were not observed in other simple epithelia, such as the liver. Electron microscopy showed that transgenic acinar cells have keratins organized in abundant filament bundles dispersed throughout the cytoplasm, in contrast to control acinar cells, which have scarce and apically concentrated filaments. The phenotype found was very similar to that reported for transgenic mice expressing a dominant-negative mutant TGF$\beta$ type II receptor (TGF $\beta$ RII mice). We show that these TGF $\beta$ RII mutant mice also have elevated K8/K18 levels. These results indicate that simple epithelial keratins play a relevant role in the regulation of exocrine pancreas homeostasis and support the idea that disruption of mechanisms that normally regulate keratin expression in vivo could be related to inflammatory and neoplastic pancreatic disorders.
\end{abstract}

J. Clin. Invest.103:1587-1595 (1999).

\section{Introduction}

Keratins are epithelia-specific intermediate-filament (IF) proteins encoded by a large multigene family; they are classified as type I or type II, based upon their charge, immunological relatedness, and sequence similarity. Because keratin filament assembly begins with the formation of a type I-type II heterodimer, all epithelial cells express at least 1 member of each keratin type (reviewed in refs. 1,2).

The cellular function of these proteins is largely unknown. Keratin expression is tightly regulated, however, both in terms of the pattern and the amount of polypeptides found in the diverse types of epithelial cells. This specific expression is thought to create different IF networks tailored to particular functions and phenotypic features. In this regard, point mutations in human epidermal and mucosal keratins, such as $\mathrm{K} 5 / \mathrm{K} 14$, $\mathrm{K} 1 / \mathrm{K} 10, \mathrm{~K} 9, \mathrm{~K} 6 / \mathrm{K} 16 / \mathrm{K} 17$, or $\mathrm{K} 4 / \mathrm{K} 13$, are associated with several inherited epithelial diseases - in particular, skin fragility diseases (reviewed in refs. 3, 4).

Keratin K8 and its partner, keratin K18, are found typically in simple or single-layered epithelial tissues (1). They are the first IF proteins to be expressed during murine embryogenesis and are found in the trophectoderm at the blastocyst stage (5-7). It has been shown recently that transgenic mice expressing a point-mutat- ed K18 keratin manifest liver inflammation and necrosis in association with hepatocyte fragility (8). These animals exhibit an increased sensitivity to toxin-induced liver injury (9). Hepatic lesions, colorectal hyperplasia and inflammation, female sterility, and embryonic lethality were also noted in K8-null mice, depending on the mouse strain $(10,11)$. These results indicate that the $\mathrm{K} 8 / \mathrm{K} 18$ pair plays an important role in the function of different simple epithelia, such as liver, gastrointestinal tract, and, possibly, reproductive tract.

Although there are several well-known pathologies that involve abnormally elevated expression of keratins - for example, high K8/K18 levels in malignant cells (12-15) - to date, the physiological consequences of the overaccumulation of keratin filaments in a cell are not clear. In transgenic mice, overexpression of other IF proteins, such as neurofilaments (expressed in neuronal cells) and vimentin (in cells of mesenchymal origin), results in morphological and functional alterations such as progressive neuronopathy resembling amyotrophic lateral sclerosis (ALS) $(16,17)$ and altered lens cell differentiation (18).

To address the importance of the correct amount of the appropriate keratin filament complement for epithelial function and development, we used transgenic mice 
to force simple epithelial cells to increase K8 expression. We reported previously that transgenic mice containing the $h k 8$ gene express the transgene in a tissue-specific, copy number-dependent fashion (19). Here we show that the lines with the higher copy number present profound structural and functional alterations in their acinar cells, eventually leading to tissue destruction and adipose atrophy of the pancreas in aging animals. We conclude that the phenotype of these animals may be relevant to understanding the origin of inflammatory and neoplastic pathologies of the exocrine pancreas.

\section{Methods}

Genomic DNA and generation of transgenic mice. Construct HK812 , containing the entire $h k 8$ gene, is described in Casanova et al. (19). Construct $3^{\prime} \Delta H K 8$ was derived from construct HK812 by deleting a $2.6-\mathrm{kb}$ XbaI-SalI fragment containing 3' flanking sequences. DNA fragments were isolated and microinjected into $\mathrm{C} 57 \mathrm{BL} / 10 \times \mathrm{BALB} / \mathrm{c}-\mathrm{F} 2$ (construct $\mathrm{HK} 8-12$ ) or C57BL/ $6 \times$ CBA-F2 (construct $3^{\prime} \Delta \mathrm{HK} 8$ ) mouse embryos, as described (19). Eight transgenic lines (TGK8-1 to TGK8-8) were obtained for the first construct and 4 lines (TGK8-9 to TGK8-12) for the second. Lines TGK8-1 to TGK8-4 correspond to lines $1864,1861,1918$, and 1865 , respectively (19). Copy number determinations were carried out as described (19), except that hybridization signals were quantified by phosphorimaging. We observed no variation in the phenotype attributable to the different genetic backgrounds used.

Mice were housed in a facility with controlled temperature, humidity, and light/dark cycles, under standard conditions. Their sanitary status was strictly controlled for both internal and external parasites, as well as for bacterial and viral infections. They were weaned at 3 weeks of age. Nontransgenic littermates were used as controls.

Preparation and analysis of RNA. Total RNA from different tissues was purified by the acid phenol method (20). K8 and K18 keratin transcripts were detected by Northern blot analysis of $10-15 \mu \mathrm{g}$ of total RNA using random-primed, ${ }^{32} \mathrm{P}$-labeled DNA probes. Probes for $h k 8$ and $m K 8$ were as described previously (19). Mouse $\mathrm{K} 18$ was detected with a $1.0-\mathrm{kbp} E c o \mathrm{RI}-P s t \mathrm{I}$ fragment of the mouse $\mathrm{K} 18 \mathrm{cDNA}$. As a loading control, the filters were stripped and rehybridized with a rat $\beta$-actin or a ribosomal $7 \mathrm{~S}$ probe.

a

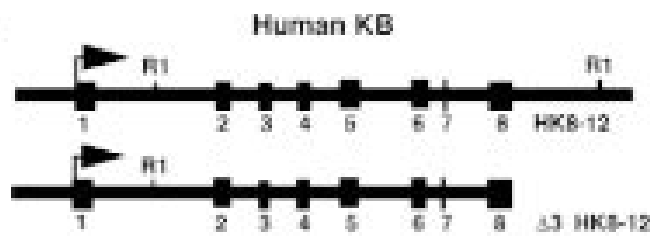

c

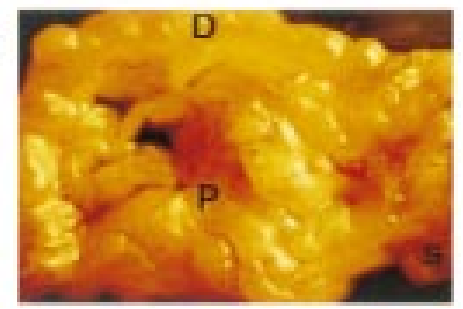

Histological and immunohistochemical analysis. For histology and immunohistochemical analysis, freshly collected tissues were fixed immediately in $10 \%$ formaldehyde or $70 \%$ ethanol, respectively, embedded in paraffin, sectioned, and stained with hematoxylin/eosin. The antibodies used were the rat mAb TROMA1 (5), which recognizes mouse and human K8; LE61, a mouse mAb that recognizes mouse K18 (21); or CAM 5.2 (Sigma Chemical Co., St. Louis, Missouri, USA), a mouse mAb that recognizes human K8. Sections were incubated with a biotinylated anti-mouse or anti-rat antibody, and then with streptavidin conjugated to horseradish peroxidase (DAKO A/S, Glostrup, Denmark). Control immunostainings using the secondary antibody in the absence of the primary antibody were routinely performed. Antibody localization was determined using 3,3diaminobenzidine (DAB) in PBS.

Enzymatic functional analysis. Fecal trypsin activity was determined as described by Fraser (22). Feces were added to $180 \mu \mathrm{L}$ sodium bicarbonate solution to bring the total to $200 \mu \mathrm{L}$, and then were placed on unprocessed autoradiographic film and incubated at $37^{\circ} \mathrm{C}$ for 1 hour. The film was then washed, and the level of gelatin emulsion degradation was measured by densitometry. Fecal fat content was stimated using the Sudan III semiquantitative-staining method (23), with 8-week-old mice fed an olive oil-rich diet for 2 days. Sudan III was added to an extension of feces, and fat drops were counted by microscopy.

Electron microscopy analysis. Pancreata from 2-month-old control and TGK8-4 transgenic mice were fixed in $2.5 \%$ glutaraldehyde in $0.1 \mathrm{M}$ phosphate buffer $(\mathrm{pH} 7.5)$ and postfixed in $1 \%$ osmium tetroxide prior to dehydration and embedding in Epon 812 resin. Semithin sections were stained with 1\% toluidine blue for field selection. Ultrathin sections were stained with uranyl acetate and lead citrate.

Protein extraction and analysis by electrophoresis and immunoblotting. Keratins from tissues and cultured cells were isolated using high-salt extractions, as described (24). Total proteins were isolated as described by $\mathrm{Ku}$ et al. (8). Protein concentrations were determined using the protein assay from Bio-Rad Laboratories Inc. (Hercules, California, USA). Loading was also confirmed by Coomassie blue staining of duplicate gels. After electrophoresis in 8.5\% SDS-PAGE gels, proteins were transferred to Immobilon-P membranes (Millipore Corp., Bedford, Massachusetts, USA). The membranes were incubated for 1 hour with rat mAb TROMA-1 (5), with M20 mouse anti-HK8 mAb (Sigma Chemical Co.), or with rabbit polyclonal anti-K18 anti-

b
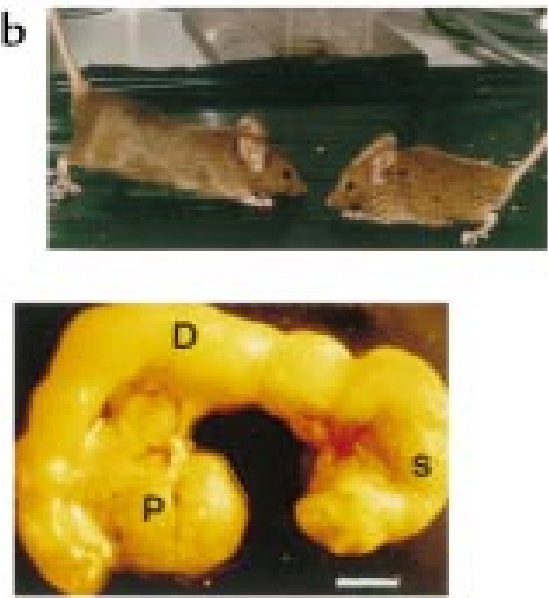

Figure 1

Phenotype of transgenic mice carrying HK8 constructs. (a) DNA constructs injected. Arrows indicate the position of the transcriptional start site; exons are denoted by filled boxes. RI represents EcoRI restriction site and is included for orientation. (b) Dwarfism of transgenic HK8 mice. Eightweek-old transgenic animal (right) and nontransgenic littermate (left). (c) Reduction of pancreas size. Pancreata from 8-week-old control (left) and transgenic mice (TGK8-4; right) were photographed. The transgenic pancreas is about $30 \%$ the size of that in nontransgenic littermates. D, duodenum; S, stomach; P, pancreas. Scale bar: $3 \mathrm{~mm}$. 
Table 1

Determination of proliferating cells by BrdU incorporation

\begin{tabular}{|c|c|c|}
\hline Mouse genotype & No. of animals ${ }^{A}$ & $\begin{array}{l}\text { Anti-BrdU-positive } \\
\text { cells per } 200 \text { cells }\end{array}$ \\
\hline Control & 5 & $2.3 \pm 0.5$ \\
\hline TGK8-8 & 5 & $22.7 \pm 3.7$ \\
\hline TGK8-4 & 4 & $14.5 \pm 2.5$ \\
\hline
\end{tabular}

AAnimals were 2-7 months old.

body 1589 (25), followed by a peroxidase-coupled anti-rat, antimouse, or anti-rabbit IgG (Jackson ImmunoResearch Laboratories, West Grove, Pennsylvania, USA). Keratin bands were viewed using enhanced chemiluminescence (ECL; Amersham Life Sciences Inc., Arlington Heights, Illinois, USA).

In vivo proliferation and apoptosis studies. For in vivo proliferation assays, control and transgenic mice received an intraperitoneal injection of bromodeoxyuridine (BrdU; $120 \mathrm{mg} / \mathrm{kg}$ body weight) (Boehringer Mannheim GmbH, Mannheim, Germany) in $0.9 \% \mathrm{NaCl}$; animals were sacrificed 2 hours later. Pancreata were fixed in $70 \%$ ethanol for 72 hours, embedded in paraffin, sectioned, and sequentially incubated for 1 hour with an antiBrdU rat mAb (kindly provided by S. Mittnacht, Chester Beatty Laboratories, London, United Kingdom) and an FITClabeled secondary anti-rat antibody (Jackson ImmunoResearch Laboratories) to label S-phase cells.

Apoptotic cells were detected using different methods. (a) Terminal deoxynucleotidyl transferase-mediated dUTP nickend labeling (TUNEL) assays (Boehringer Mannheim GmbH) were performed following the manufacturer's instructions. (b) Analyses of hematoxylin/eosin- and toluidine blue-stained semithin sections from pancreata of 2- to 7-month-old mice were performed. Cells displaying nuclear and cytoplasmic condensation or nuclear/cellular fragmentation were considered apoptotic. (c) Nuclei of pancreas sections were stained as follows: ethanol-fixed histological preparations were deparaffinized, treated with RNase A $(200 \mu \mathrm{g} / \mathrm{mL})$ for 30 minutes at $37^{\circ} \mathrm{C}$, washed, mounted with Micromount (Surgipath Canada
Inc., Winnipeg, Manitoba, Canada) containing propidium iodide (PI; $50 \mu \mathrm{g} / \mathrm{mL}$ ), and then examined by confocal microscopy. Cells with condensed chromatin or nuclear fragmentation were considered apoptotic. (d) DNA content was determined by flow cytometry. Nuclei were isolated by homogenizing pancreata in a buffer containing $10 \mathrm{mM}$ Tris ( $\mathrm{pH}$ 7.4), $10 \mathrm{mM} \mathrm{NaCl}, 3 \mathrm{mM} \mathrm{Cl}_{2} \mathrm{Mg}, 0.1 \mathrm{mM}$ PMSF, and 5\% NP40, followed by centrifugation at $400 \mathrm{~g}$ for 10 minutes. The nuclear pellet was resuspended, fixed in ethanol, treated with RNase A $(200 \mu \mathrm{g} / \mathrm{mL})$ citrate buffer, and stained with PI $(50 \mu \mathrm{g} / \mathrm{mL})$, according to Darzynkiewicz and Juan (26). DNA content was analyzed in an Epics XL flow cytometer (Coulter Electronics Ltd., Hialeah, Florida, USA). For each analysis, a total of 10,000 nuclei were analyzed. Off-line analysis was done using the WinMDI version 2.7 software (a kind gift of J. Trotter, Scripps Research Institute, La Jolla, California, USA). Integrated and peak DNA signals were used for aggregate discrimination. To avoid cellular debris, events were gated out 1 log below the 2-N DNA peak. The number of BrdU- and TUNEL-positive cells were scored in a blind fashion by 2 persons.

\section{Results}

Generation of HK8 transgenic mice. We previously reported the generation of 4 transgenic lines (TGK8-1 to TGK84) that contain the unrearranged HK8-12 construct (Figure 1a). As a result of further microinjections, we obtained 4 additional lines expressing this construct (TGK8-5 to TGK8-8) and 4 lines expressing construct

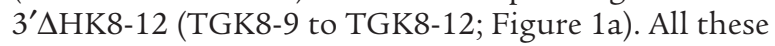
lines express human $\mathrm{K} 8$ (HK8) mRNA proportionally to the integrated transgene copy number and in a tissuespecific pattern similar to the endogenous mouse K8 $(m K 8)$ gene (ref. 19 and results not shown).

Mice carrying more than 17 transgene copies (hemizygous TGK8-4, TGK8-8, TGK8-12, and homozygous TGK8-11) had somewhat erect hair and reduced growth (Figure 1b); their weight was approximately $70 \%$ that of
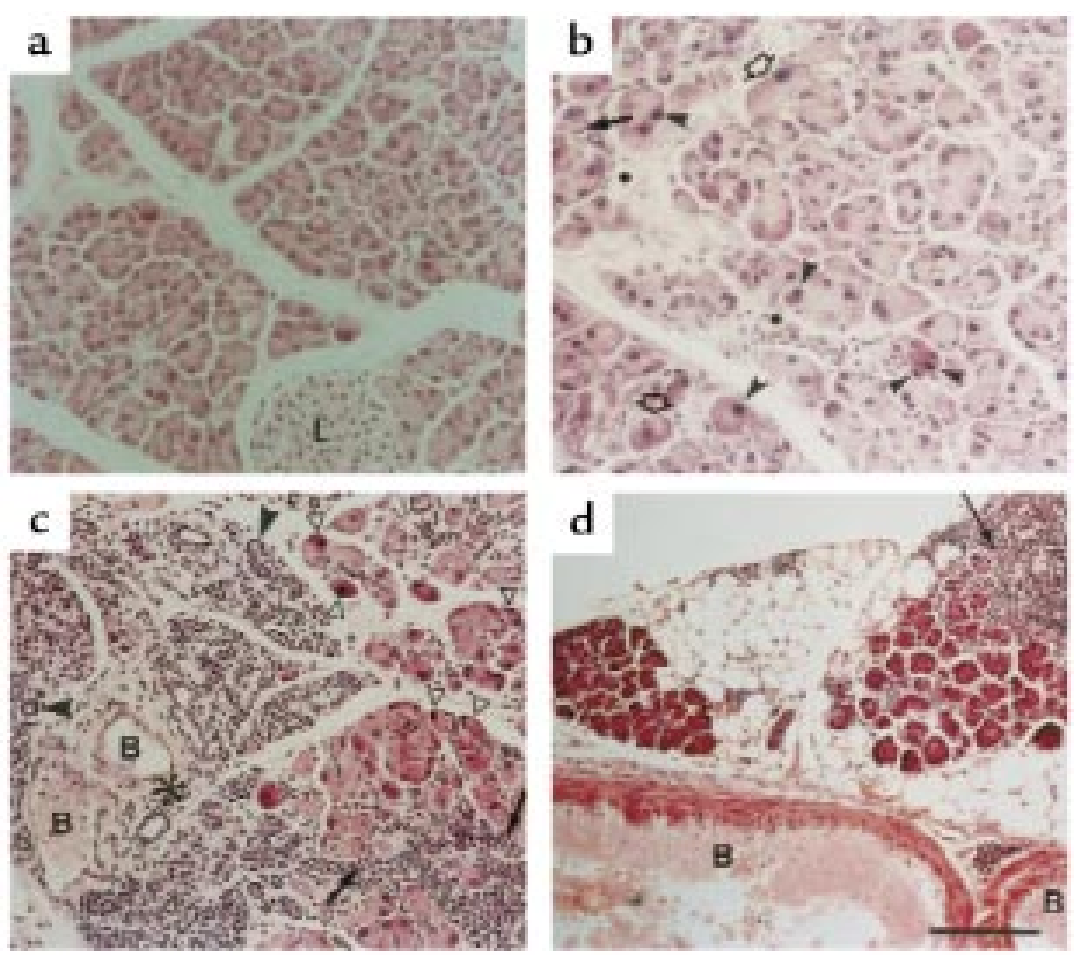

\section{Figure 2}

Pancreatic histopathology of HK8 transgenic mice. Pancreas sections from 2-month-old (a-c) and 1-year-old (d) animals stained with hematoxylin/eosin. (a) Control pancreas; (b) TGK8-8; (c and $\mathbf{d}$ ) TGK8-4. (a and $\mathbf{b}$ ) Loss of normal exocrine pancreas architecture and dysplastic changes in transgenic acinar cells. Arrowheads denote apoptotic figures; the arrow depicts diffuse mononuclear inflammatory infiltrate cells; asterisks show intralobular sclerosis; open arrows denote dysplastic cells with enlarged cellular and nuclear size. (c and $\mathbf{d}$ ) Pancreata from TGK8-4 mice. (c) Extensive metaplasia of acinar cells, giving rise to aberrant ductules (arrowheads). A diffuse mononuclear inflammatory infiltrate (arrows), interlobular sclerosis (asterisk), and apoptotic cells (open triangles) were also frequent. (d) Replacement of acinar tissue by adipose cells, presence of a perivascular mononuclear infiltrate (arrow), and eosinophilic foci of acinar cells (oval structures) observed in aging mice. L, islets of Langerhans; B, blood vessels. Scale bar: (a and b) $100 \mu \mathrm{m}$; (c) $125 \mu \mathrm{m}$; (d) $200 \mu \mathrm{m}$. 


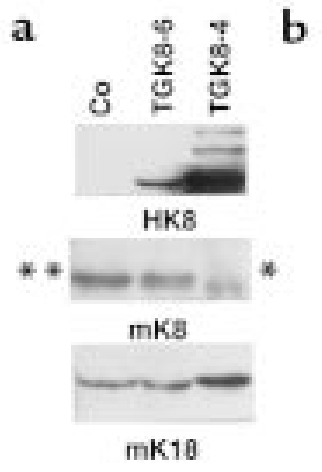

Figure 3

Analysis of HK8 protein and mRNA in transgenic mouse pancreas. (a) Western blot analysis of total protein extracts $(10 \mu \mathrm{g})$ from pancreata of 6-week-old nontransgenic mice (Co), nonphenotypic transgenic mice (line TGK8-6), and phenotypic transgenic (line TGK8-4) mice. Triplicate gels were immunoblotted using anti-HK8 mAb M20 (top); rat mAb TROMA-1, which, under these conditions, recognizes mouse K8 strongly (double asterisk) and human K8 weakly (single asterisk) (middle); or the anti-K18 polyclonal antibody 1589 (bottom). (b) Northern blot analysis using $15 \mu \mathrm{g}$ of total RNA from pancreata of 6-week-old animals bearing different $h k 8$ transgene copy numbers. The filters were sequentially hybridized for HK8 (top), mK18 (middle), and 7S (bottom). (c) Coomassie blue-stained gel. Equal amounts of cytoskeletal extracts from the same amount (in grams) of pancreata and livers from 6-weekold control and transgenic mice were loaded. (d and $\mathbf{e})$ Immunodetection of K8 in pancreas sections. Paraffin-embedded sections from 2-month-old control (d) and transgenic (e) pancreata. Control pancreas was stained with the TROMA-1 mAb to view endogenous mK8. The signal is restricted to the apical region of acinar cells and to ductal cells. Transgenic pancreas sections were labeled with the HK8specific CAM 5.2 antibody. The staining is stronger and appears throughout the cytoplasm of acinar cells. The inset shows an area of ductules reacting with antibody CAM 5.2; the arrow depicts an acinus undergoing dedifferentiation into a tubular complex. L, islet of Langerhans. Scale bar: $60 \mu \mathrm{m}$.
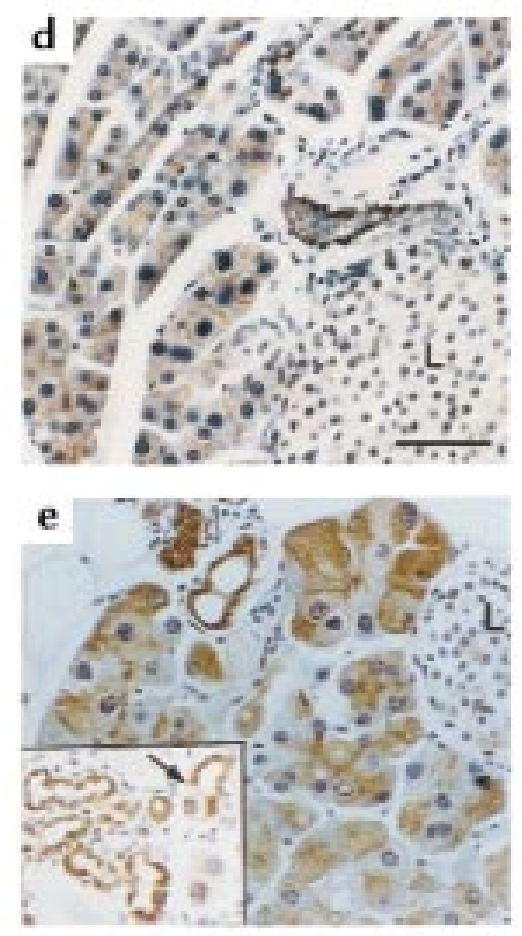

nontransgenic littermates. The life span of these mice was normal, although we occasionally observed early mortality in some hemizygous animals. When hemizygous mice of lines TGK8-4 and TGK8-8 were mated, very few homozygous mice were born, and most of these died within a few days of birth. Unless otherwise specified, the results that follow were obtained in hemizygous animals from these 2 lines, the most extensively studied.

HK8 transgenic mice have exocrine pancreas alterations. To determine the cause(s) underlying the dwarf phenotype, 8 -week-old transgenic mice and nontransgenic littermates were dissected. The only remarkable macroscopic difference found was a dramatic reduction in the size of the transgenic pancreas, whose weight was only approximately $30 \%$ that of the nontransgenic mouse pancreas (Figure $1 \mathrm{c}$ and results not shown).

Histological examination of the pancreas sections showed that the exocrine component was altered (Figure 2). The abnormalities were more severe and occurred at an earlier age in line TGK8-4 than in line TGK8-8, although in adulthood ( 7 months) both lines developed the same lesions. The acini had lost their typical lobular organization of acinar cells, with the apical region oriented toward the central lumen (Figure 2, compare $\mathrm{a}$ and $\mathrm{b}$ ). In addition, as a consequence of acinar cell redifferentiation, a large part of the acinar lobules were replaced by ductal cells, resulting in extensive areas of tubular complex appearance (Figure 2 c; see also inset in Figure 3e). Acinar cells were also observed showing dysplastic changes characterized by enlarged cellular and nuclear size, cellular pleomorphism, and loss of the basal nuclear polarity typical of normal aci- nar cells (Figure 2b). The number of these cells increased with age (results not shown). Intra- and interlobular fibrosis was also seen (Figure 2, b and c). Inflammation was present as diffuse mononuclear infiltrates in the conjunctive stroma of pancreata in young animals (Figure 2, b and c), a pathology reminiscent of human chronic pancreatitis. A progressive substitution of acinar epithelium by adipose tissue (adipose atrophy) was observed, as well as the presence of persistent focal infiltrates of mononuclear inflammatory cells around isolated interacinar ducts and blood vessels (Figure 2d). Eosinophilic foci of acinar cells, considered a focal hyperplasia marker in rats (27), were observed in the pancreata of phenotypic mice at this age (Figure $2 \mathrm{~d}$ ).

To determine whether these histological abnormalities affected pancreatic function, we assayed fecal trypsin activity (22) and found that it was drastically reduced in transgenic mice (30-50 times in TGK8-8 and at least 100 times in TGK8-4 mice; results not shown). We also found abundant fat drops (steatorrhea) in feces of transgenic mice fed a fat-enriched diet, indicating fat malabsorption as a consequence of lipase deficiency. Together, these results show that HK8 mice have a severe pancreatic insufficiency.

Although loss of normal acinar architecture and dysplasia of the exocrine pancreas are characteristic of a preneoplastic state $(28,29)$, we have not observed pancreatic neoplasia in transgenic mice under 2 years of age. Only the exocrine pancreas seemed to be affected, because the islets of Langerhans and other simple epithelia, such as liver, lung, intestine, and stomach, showed no histological differences between control and transgenic mice. 

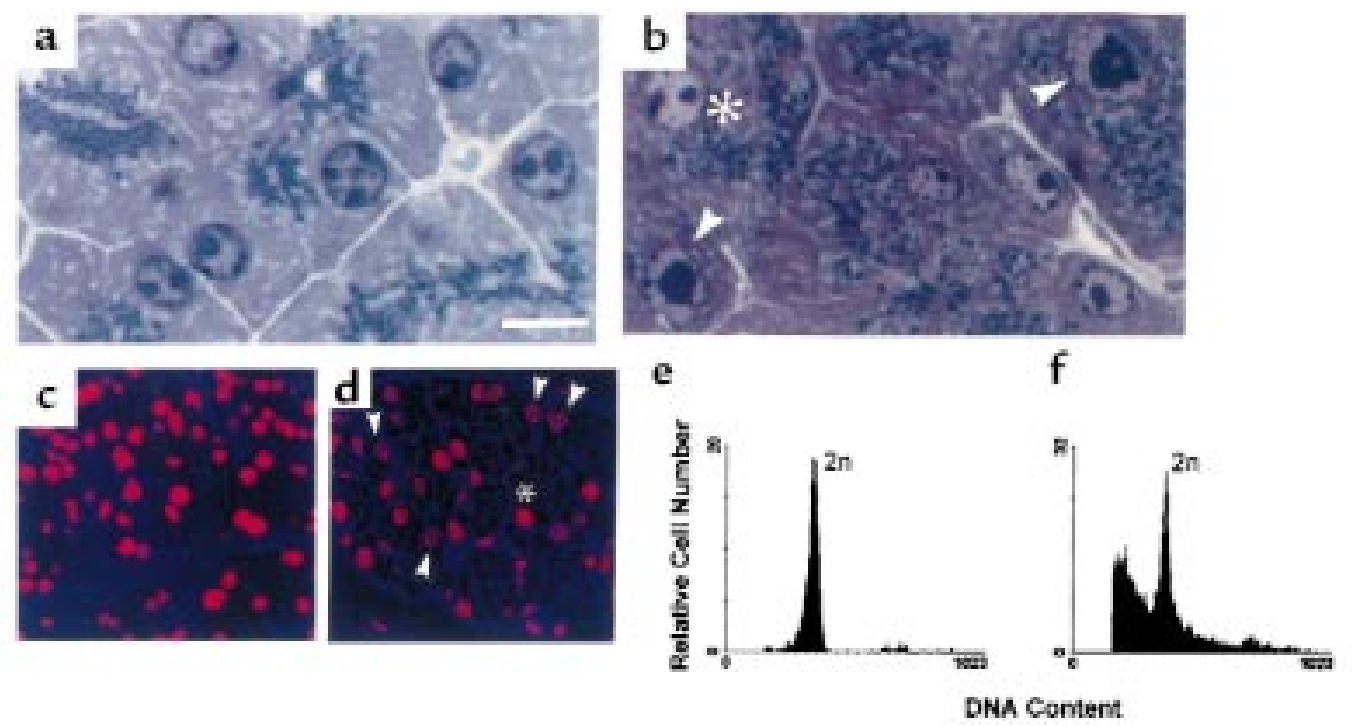

Figure 4

Increased apoptosis in the transgenic pancreas. ( $\mathbf{a}$ and $\mathbf{b})$ Toluidine blue staining of semithin sections $(1 \mu \mathrm{m})$. (c and $\mathbf{d})$ Confocal microscopy of PIstained nuclei. (e and $\mathbf{f}$ ) DNA content determination by flow cytometry. Integrated and peak DNA signals were used for aggregated discrimination. To avoid cellular debris, events were gated out 1 log below the 2-N DNA peak. (a, c, and e) Control pancreas; (b, d, and f) transgenic pancreas. Arrowheads and asterisks denote apoptotic and normal nuclei, respectively. Scale bar: (a and b) $10 \mu \mathrm{m}$; (c and d) $30 \mu \mathrm{m}$.

Regarding the time at which pancreatic lesions appear, exocrine cells appeared normal both in 15.5-day postcoitum embryos and newborn transgenic mice, indicating that the changes described develop after birth. In TGK84 mice, histological anomalies were first detected in 2week-old animals, when weight differences between control and transgenic mice also become apparent (not shown). At this time, pancreas abnormalities were limited to some disorganization of the acinar lobules. The acinarductal conversion was first detected in 3-week-old animals and was clearly present 1 week later. The adipose conversion took place only in adult animals (2-4 months old; results not shown).

To test whether dwarfism was hormonal in origin, we measured hypophyseal and thyroid hormone levels, because of their well-known implication in animal development and because of the HK8 expression in hypophyseal and thyroid glands. No statistically significant differences were found between control and transgenic mice in the amount of circulating T4, T4 and T3 in the liver or brain, or $\mathrm{T} 4$ and $\mathrm{T} 3$ contents in the thyroid gland, either incorporated into protein or ready for secretion ("free fraction"); nor were there differences in thyroid weight or in pituitary thyroid-stimulating hormone (TSH) or growth hormone (GH) contents (results not shown).

Pancreatic lesions correlate with increased keratin levels. Western blot analysis of total protein extracts $(10 \mu \mathrm{g}$; Figure $3 \mathrm{a})$ or enriched cytoskeletal fractions (results not shown) demonstrated that transgenic pancreata expressed human $\mathrm{K} 8$, because they contained a $42.5-\mathrm{kDa}$ protein that reacted specifically with an anti-HK8 antibody (Figure 3a, top) and comigrated on SDS-PAGE gels with the band corresponding to HK8 from HeLa cells (result not shown). In line TGK8-4 pancreata, HK8 was strongly expressed, as compared with nonphenotypic transgenic mice (Figure $3 \mathrm{a}$, top; compare the intensity of the band corresponding to TGK8-4 with that of TGK8-6. This radiograph was overexposed to show that control pancreata were completely negative for HK8). As a result of the presence of transgenic HK8, endogenous $\mathrm{mK} 8$ was concomitantly reduced or absent (Figure 3a, middle), whereas, in contrast, the amount of mK18 (the partner of K8) was increased (Figure 3a, bottom). Similar changes in the amount of pancreatic cytoskeletal proteins were also observed in Coomassie blue-stained gels of purified IF proteins (Figure 3c). By densitometric analysis, the amount of K8/K18 was estimated to be approximately 3fold higher in phenotypic mice than in nontransgenic or nonphenotypic transgenic mice.

We found that the increase in $\mathrm{K} 18$ also occurred at the mRNA level (Figure 3b). In pancreata from mice progressively overexpressing transgenic HK8 mRNA (lines TGK8-3, TGK8-8, and TGK8-4; Figure 3b, top), the amount of mK18 mRNA increases concomitantly (Figure $3 \mathrm{~b}$, middle). A similar increase in mK18 mRNA was also found in liver, stomach, and thymus from these mice (results not shown).

The relationship between morphological changes and transgene expression at the cellular level was studied by immunohistochemical staining. Control pancreas sections stained with TROMA-1, which detects endogenous $\mathrm{K} 8$, gave a weak signal, localized mainly at the apical region of the acinar cells (Figure 3d) and in ductal cells. Transgenic pancreas sections treated with the HK8-specific mAb CAM 5.2 showed strong, filamentous staining throughout the cytoplasm of acinar and ductal cells (Figure 3e). Similarly, immunostaining experiments using the LE61 mAb showed that whereas mK18 is barely detected in the nontransgenic pancreas, a prominent $\mathrm{mK} 18$ signal was detected in acinar and ductal cells of transgenic mice (results not shown). Collectively, these biochemical and immunohistochemical results demonstrate a correlation between the alterations observed in transgenic mouse acinar cells and the presence in these cells of increased 


\section{Figure 5}

Ultrastructural analysis of pancreatic acinar transgenic cells. (a and b) Low-magnification electron microscopy survey of control (a) and TGK8-4 (b) acinar cells from 2-month-old littermates. Arrowheads mark the lumen in the acini of control cells. Note the accumulation and loss of the apical localization of zymogen granules in transgenic cells. Apoptotic transgenic cells with condensed chromatin, indented nuclei, and swollen endoplasmic reticulum are also frequent. (c) Electron micrograph showing the apical region of 2 cells around the acinar lumen in the control pancreas. IFs, frequently anchored to desmosomes, are scarce and are restricted to this region in these cells. ( $\mathbf{d}$ and $\mathbf{e})$ Bundles of IFs are abundant and found dispersed throughout the cytoplasm in transgenic acinar cells. S, secretory granules; $D$, desmosome. Scale bar: (a and b) $5 \mu \mathrm{m}$; (c-e) $250 \mathrm{~nm}$.

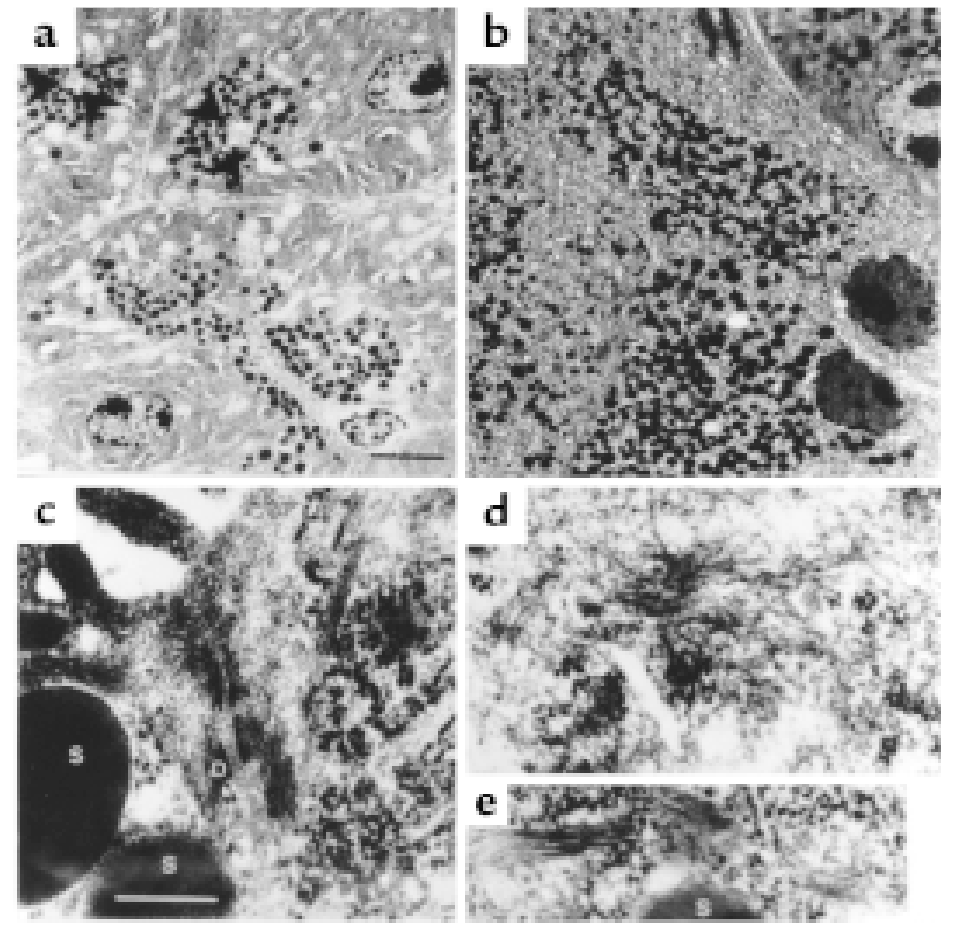

amounts of K8 and K18 keratins organized in a cytoskeleton dispersed throughout the cytoplasm, in contrast to the lower amounts and apical localization of these keratins in acinar cells from control animals.

Acinar cells from transgenic pancreata show increased proliferation and apoptosis. DNA synthesis was measured in the pancreata of 2- to 7-month-old mice using an anti-BrdU antibody to estimate BrdU incorporation. The number of acinar cells synthesizing DNA was 7-11 times higher in transgenic mice (Table 1). We also analyzed apoptosis in pancreas sections of 2- to 7-month-old control and transgenic mice (from both TGK8-4 and TGK8-8) by different methods. Using the TUNEL assay, we found that apoptosis was clearly increased in transgenic animals (Table 2). Similarly increased numbers of apoptotic cells were also detected in transgenic pancreas sections stained with hematoxylin/eosin (Figure 2, b and c) and in semithin sections stained with toluidine blue (Figure 4, a and b). A high proportion of acinar cells with fragmented or perinuclearly collapsed chromatin were also detected by confocal microscopy of PI-stained transgenic pancreas sections (Figure 4, c and d). Finally, we also determined the number of pancreas nuclei with hypodiploid DNA content using flow cytometry. In control animals, $2.46 \%$ of the total events counted had hypodiploid DNA, whereas this proportion increased to $35.03 \%$ and to $32.78 \%$ in TGK8-4 and TGK8-8 mice, respectively (Figure 4, e and f; and data not shown).

These results show that, in addition to the alterations in differentiation, increased keratin expression also disturbs the processes of proliferation and apoptosis in acinar cells.

Ultrastructural studies. Normal acinar cells contain secretory granules (zymogen) concentrated at the apical region, as seen in toluidine blue-stained semithin sections (Figure 4a). In contrast, acinar cells of pathological pancreata showed a larger number of zymogen granules dispersed throughout the cytoplasm (Figure 4b).
These results were corroborated by electron microscopy studies (Figure 5, a and b). These pictures also show that transgenic acinar cells frequently manifest nuclei that are fragmented or that have indented profiles and greater electron density than control nuclei, in conjunction with a swollen endoplasmic reticulum, characteristics of apoptotic cells. At higher magnifications, control acinar cells showed few IFs, and these were mainly anchored to desmosomes at the apical region of the cytoplasm (Figure 5c). In contrast, transgenic acinar cells had abundant filament bundles of normal appearance, located not only at the secretory apical region but also dispersed throughout the cytoplasm (Figure 5, d and e). These results corroborate the immunostaining data (Figure 3) and demonstrate that the increased amounts of keratin IF, containing both the transgenic and the endogenous proteins, were properly assembled from a structural point of view, although the resultant cytoskeleton was more uniformly distributed throughout the cytoplasm than in normal cells.

Increased $K 8 / K 18$ levels are also found in the altered exocrine pancreata of transgenic mice expressing a mutant TGF- $\beta$ type II receptor. Analyses of the exocrine pancreata of transgenic mice expressing a dominant-negative mutant form of the TGF- $\beta$ type II receptor, generated by Böttinger et al. (30), showed that these animals manifest a pancreatic phenotype very similar to those described above, including reduced organ weight, acino-ductular metaplasia, fibrosis, and adipose replacement, as well as increased proliferation and apoptosis (30). As observed in HK8 mice, these alterations also appear progressively with age in TGF $\beta$ RII mice.

In view of these similarities, we asked if TGF $\beta$ RII mice also have altered K8/K18 levels. Immunohistochemical analysis using anti-K8 and anti-K18 antibodies (Figure 6 , a and b; and results not shown), as well as Western blots and Coomassie blue-stained gels (Figure 6c), 


\section{Figure 6}

Analysis of $\mathrm{K} 8$ and $\mathrm{K} 18$ protein expression in pancreata from transgenic TGF $\beta$ RII mice. (a and b) Immunodetection of K8. Paraffinembedded sections from control (a) and transgenic (b) pancreas sections from 6month-old littermates were stained with TROMA-1 antibody. Scale bar: $50 \mu \mathrm{m}$. (c) Western blot analysis of $\mathrm{K} 8$ and $\mathrm{K} 18$ in TGF$\beta$ RII mice (TG) and control nontransgenic mice (Co) (top). Total protein extracts (10 $\mu \mathrm{g})$ from pancreata of 4-month-old control and transgenic mice (which presented a moderate phenotype) were blotted with antibodies TROMA-1 (recognizing K8) and 1589 (recognizing K18). Note the increased level of these keratins in TGF $\beta$ RII mice, also detected in Coomassie blue-stained gels of cytoskeletal fractions (bottom).

demonstrated elevated levels of these 2 keratins in the acinar cells of these mice. These data were indistinguishable from those found in the same cells of HK8 mice processed in parallel, both in terms of the homogeneity of cytoplasmic distribution and signal intensity (results not shown; compare Figures 3 and 6). These results show that TGF- $\beta$ signaling and the amount of keratin $\mathrm{K} 8$ and $\mathrm{K} 18$ in acinar cells are related in a presently unknown manner, such that decreased signaling due to loss of receptor function leads to increased keratin levels. They also confirm that there is a correlation between the presence of elevated K8/K18 levels and the described type of acinar alterations.

Tissue specificity of the phenotype. The $b k 8$ transgene follows the expression pattern of the endogenous $m K 8$ (19), although pathological changes were observed exclusively in the exocrine pancreas. To determine whether this is because of tissue-specific differences in transgene expression, we studied HK8 expression levels in the liver at the protein and mRNA level (Figure $3 \mathrm{~b}$ and results not shown). The results obtained were similar to those shown in Figure 3 for the pancreas. From the biochemical point of view, therefore, there is no obvious explanation for the tissue specificity of the phenotype.

A similar phenomenon is also found in TGFßRII mice. Although these animals express comparably high levels of the dominant-negative receptor form in several simple epithelia, and TGF- $\beta$ signaling was inhibited in the pancreas and liver, histological alterations were found only in acinar cells (30). This coincidence reinforces the conclusion that in HK8 and TGFßRII mice, the phenotype is due to the increase in K8/K18. Given that the pancreas appears unaltered in K8-deficient mice (11), this indicates a particular sensitivity of acinar cells to

Table 2

Determination of apoptotic cells by TUNEL analysis

\begin{tabular}{ccc}
\hline Mouse genotype & No. of animals & Apoptotic cells $/ 300$ cells \\
Control & 5 & $0.5 \pm 0.5$ \\
TGK8-8 & 6 & $18.4 \pm 9.1$ \\
TGK8-4 & 4 & $21.7 \pm 9.7$ \\
\hline
\end{tabular}

ATwo-to-seven month old. increased keratin levels, which could be relevant in the etiology of inflammatory and neoplastic diseases.

\section{Discussion}

Keratins play an essential role in regulation of growth and differentiation in the exocrine pancreas. We have demonstrated that HK8 expression in transgenic mice leads to increased keratin IF levels in simple epithelia and induces severe alterations of the exocrine pancreas, including dysplasia and dedifferentiation of acinar cells, ductal metaplasia, fibrosis, inflammation, and adipose conversion (Figure 2). These alterations appear progressively and are first detected in the second week of life, coinciding with the observed decrease in the growth rate of the transgenic mice at this age. Transgenic mice exhibiting a weak or moderate phenotype had very low fecal trypsin and lipase levels, suggesting that the smaller size of these animals has a nutritional basis due to pancreatic insufficiency. Nonetheless, normal amounts of amylase (detected both by Northern blot and immunohistochemical staining; data not shown) and zymogen granules (Figures 4 and 5) were found in these mice, indicating that synthesis of pancreatic enzymes is not substantially perturbed. Pancreatic insufficiency may thus be due to a defect in the processing and/or secretion of these granules, consistent with the observed anomalous distribution of zymogen granules, which are dispersed throughout the cytoplasm of transgenic acinar cells rather than being concentrated at the apical pole (Figures 4 and 5). Microtubules and microfilaments are reported to be involved in the polarized vesicular transport and the exocytosis of zymogen granules (31-34). Our results, which show a correlation between the loss of the apical localization of keratin filaments and the cytoplasmic dispersion of zymogen granules in transgenic acinar cells, suggest that simple epithelial keratins may also have a relevant function in these processes.

The physiological relevance of these results is reinforced by the finding that transgenic mice expressing a mutant form of the TGF- $\beta$ type II receptor, which blocks signaling by all 3 TGF- $\beta$ isoforms, develop a phenotype extremely similar, if not identical, to that of HK8 mice in their histopathological characteristics, time of onset, and tissue specificity (30). From these animals, it has been concluded that TGF- $\beta$ negatively controls growth of aci- 
nar cells and is essential for the maintenance of a differentiated acinar phenotype in the exocrine pancreas. We found that TGF $\beta$ RII mice, exactly as HK8 mice, have elevated levels and homogeneous cytoplasmic distribution of K8/K18 in acinar cells (Figure 6). These results strongly suggest that these keratins are involved in the TGF- $\beta$ signaling mechanism, a possibility that we are presently investigating. The function of keratins has been largely unknown, although they have been related to providing resistance for cells $(3,4,8)$. We recently demonstrated that epidermal keratins K10 and K16 are involved in the control of cell proliferation and differentiation in vitro (35); the data presented here extend this function to simple keratins in vivo. This involvement is also supported by the observation that K8/K18 bind members of the 14-33 protein family (known to interact with a number of kinases and phosphates) and bind to the heat shock protein 70 (HSP-70), and a protein kinase $\mathrm{C} \varepsilon$-related kinase (reviewed in ref. 7).

Implications for buman disease: chronic pancreatitis and pancreatic cancer. There appears to be some consistency in the response of the pancreas to a variety of insults. Tubular complex formation, interstitial fibrosis, and mucoid cell metaplasia have all been associated, to some extent, with ductal obstruction-induced atrophy, adenocarcinoma, and chronic pancreatitis (36-38). All these characteristics are present in HK8 mice. Aging HK8 mice show adipose atrophy of the pancreas and chronic inflammation, also present in human chronic pancreatitis. These animals, therefore, recapitulate many features characteristic of human chronic pancreatitis and may constitute a valuable model for this disease.

Our results may also have implications for pancreatic carcinogenesis. The morphological aspect of most exocrine pancreas cancers is reminiscent of ductal cells, as has long been recognized by pathologists $(36,39)$, although ductal cells constitute a minor cell fraction in normal tissue. In addition, pancreatic cancer cells express differentiation markers characteristic of ductal cells (40). When human exocrine pancreas cells are cultured in vitro, a phenotypic switch takes place from acinar to ductal characteristics (41), which is reversible upon cessation of growth (42). Because preneoplastic lesions have not been clearly identified in the human pancreas, considerable controversy exists on the acinar or ductal origin of pancreatic cancers (43).

The first signs of anomaly in HK8 mice are loss of acinar architecture, acinar cell dysplasia, and increased proliferation, all characteristics of early neoplastic stages; they later undergo acino-ductal dedifferentiation. These features support the hypothesis that acinar cells are, in fact, the origin of pancreatic cancer and that they dedifferentiate to the ductal phenotype observed in most tumors during the carcinogenic process (43, 44). Transgenic mice expressing oncogenes (c-myc and SV40 T antigen; refs. 28, 45) and growth factors (TGF$\alpha$; refs. 46-48), or that are deficient in TGF- $\beta$ signaling (30) in acinar cells, also reinforce this model. These characteristics suggest that the HK8 mouse pancreas could represent the early stages in the pathway to pancreatic neoplasia. That these lesions do not progress to neoplasia may be a consequence of the elevated apop- tosis observed and an indication of the requirement for additional defects in other genes (e.g., p53) for carcinogenesis to proceed. Carcinogenesis experiments are currently under way, and the HK8 mice are being crossed with $p 53$-null mice to test this hypothesis.

Other studies also suggest the relevance of keratins $\mathrm{K} 8 / \mathrm{K} 18$ in carcinogenesis; for instance, these keratins are involved in aspects of tumorigenic cell behavior, such as cell migration and invasiveness (49-51). It has also been reported that $\mathrm{K} 8 / \mathrm{K} 18$ are induced by $\mathrm{v}$-ras in transformed epidermal keratinocytes $(52,53)$ and that there is a positive correlation between the expression of these keratins and epidermal cell malignancy both in vitro $(54)$ and in vivo $(12,13)$. In transitional cell carcinoma, increased K8 and K18 levels have been detected at the tumor invasion front, and there is a relationship between the expression of these keratins and tumor malignancy $(14,15)$. It is thus possible that, under certain circumstances, K8/K18 may provide a permissive cellular environment for induction of malignancy.

These data, together with those from our transgenic mice, suggest that a relationship exists between deregulated K8/K18 expression and disease, and that the elucidation of the cellular and molecular mechanisms by which HK8 mice develop pathological features in pancreatic acinar cells could contribute to our understanding of the etiology of inflammatory and neoplastic human diseases.

\section{Acknowledgments}

We thank Werner Franke, Rolf Kemler, E. Birgit Lane, Carlos Caulin, R.G. Oshima, Lalage M. Wakefield, and Sybille Mittnacht for providing materials, and Francisco X. Real for discussion and advice. We are particularly grateful to Isabel Fabregat, Alberto Alvarez, and Jose Carlos Segovia for their expert help in the analysis of apoptosis; to Jesús Martínez-Palacios for mouse husbandry; to Claudia Pérez and M. Isabel de los Santos for invaluable assistance in histology and immunostaining; to Soledad Moreno for photographic work; and to C. Mark for expert editorial assistance. This work was supported in part by grant PB 94-1230 of the Spanish Dirección General de Investigación Científica y Tecnológica.

1.Moll, R., Franke, W.W., and Schiller, D. 1982. The catalog of human cytokeratins: patterns of expression in normal epithelia, tumors and cultured cells[review]. Cell. 31:11-24.

2. Fuchs, E., and Weber, K. 1994. Intermediate filaments: structure, dynamics, function and disease. Annu. Rev. Biochem. 63:345-382.

3. McLean, W.H., and Lane, E.B. 1995. Intermediate filaments in disease[review]. Curr. Opin. Cell Biol. 7:118-125.

4. Fuchs, E. 1996. The cytoskeleton and disease: genetic disorders of IFs. Annu. Rev. Genet. 30:197-231.

5. Brûlet, P., Babinet, C., Kemler, R., and Jacob, F. 1980. Monoclonal antibodies against trophectoderm-specific markers during mouse blastocyst formation. Proc. Natl. Acad. Sci. USA. 77:4113-4117.

6. Jackson, B.W., Grund, C., Winter, S., Franke, W.W., and Illmensee, K. 1981. Formation of cytoskeletal elements during mouse embryogenesis: epithelial differentiation and intermediate-sized filaments in early postimplantation embryos. Differentiation. 20:203-216.

7. Oshima, R.G., Baribault, H., and Caulín, C. 1996. Oncogenic regulation and function of keratins 8 and 18. Cancer Metastasis Rev. 15:445-471.

8. Ku, N.O., Michie, S., Oshima, R.G., and Omary, M.B. 1995. Chronic hepatitis, hepatocyte fragility, and increased soluble phosphoglycokeratins in transgenic mice expressing a keratin 18 conserved arginine mutant. J. Cell Biol. 5:1303-1314.

9. Ku, N.O., et al. 1996. Susceptibility of hepatotoxicity in transgenic mice that express a dominant-negative human keratin 18 mutant. J. Clin. Invest. 98:1034-1046.

10. Baribault, H., Price, J., Miyai, K., and Oshima, R.G. 1993. Mid-gestation- 
al lethality in mice lacking keratin 8. Genes Dev. 7:1191-1202.

11. Baribault, H., Penner, J., Iozzo, R.V., and Wilson-Heiner, M. 1994. Colorectal hyperplasia and inflammation in keratin 8-deficient FVB/N mice. Genes Dev. 8:2964-2973.

12. Markey, A.C., et al. 1991. Expression of simple epithelial keratins 8 and 18 in epidermal neoplasia. J. Invest. Dermatol. 97:763-770.

13. Larcher, F., et al. 1992. Aberrant expression of the simple epithelial type II keratin 8 by mouse skin carcinomas but not papillomas. Mol. Carcinog. 6:112-121.

14. Schaafsma, H.E., et al. Cytokeratin expression patterns in metastatic transitional cell carcinoma of the urinary tract. An immunohistochemical study comparing local and autologous metastases. Am. J. Pathol. 1389-1400.

15. Schaafsma, H.E., et al. 1993. Increased expression of cytokeratins 8, 18 and vimentin in the invasion front of mucosal squamous cell carcinoma. J. Pathol. 170:77-86.

16. Xu, Z., Cork, L.C., Griffin, J.W., and Cleveland, D.W. 1993. Increased expression of neurofilament subunit NF-L produces morphological alterations that resemble the pathology of human motor neuron disease. Cell. 73:23-33.

17. Côte, F., Collard, F.J., and Julien, J.P. 1993. Progressive neuropathy in transgenic mice expressing the human neurofilament heavy gene: a mouse model of amyotrophic lateral sclerosis. Cell. 73:35-47.

18. Capetanaki, Y., Smith, S., and Heath, J.P. 1989. Overexpression of the vimentin gene in transgenic mice inhibits normal lens cell differentiation. J. Cell Biol. 109:1653-1664.

19. Casanova, L., et al. 1995. Tissue-specific and efficient expression of the human simple epithelial keratin 8 gene in transgenic mice. J. Cell Sci. 108:811-820.

20. Chomczynski, P., and Sacchi, N. 1987. Single-step method of RNA isolation by acid guanidinium thiocyanate-phenol-chloroform extraction. Anal. Biochem. 162:156-159.

21. Lane, E.B. 1982. Monoclonal antibodies provide specific intramolecular markers for the study of epithelial tonofilament organization. J. Cell Biol. 92:665-673.

22. Fraser, C.M. 1988. Valores y procedimientos químicos. In Manual de Merck de Veterinaria. Tercera edición. C.M. Fraser et al., editors. Centrum. Barcelona, Spain. 1026-1027.

23. Nelson D.A., Tomar, R.H., and Washington, J.A. 1988. Examen microscópico de las heces. In Todd-Sanford-Davidsohn Diagnóstico y tratamiento clínicos por el laboratorio. D.A. Nelson, J.A. Washington, W.W. McLendon, B.E. Statland, and R.H. Tomar, editors. Salvat Editores S.A. Barcelona, Spain. 710 pp.

24. Achtstaetter, T., Hatzfeld, M., Quinlan, R.A., Parmelee, D.C., and Franke, W.W. 1986. Separation of cytokeratin polypeptides by gel electrophoretic and chromatographic techniques and their identification by immunoblotting. Methods Enzymol. 134:355-371.

25. Oshima, R.G. 1981. Identification and immunoprecipitation of cytoskeletal proteins from extraembryonic endodermal cells. J. Biol. Chem. 256:8124-8133.

26. Darzynkiewicz, Z., and Juan, G. 1997. DNA content measurement for DNA ploidy and cell cycle analysis. In Current protocols in cytometry. J.P. Robinson. et al., editors. John Wiley \& Sons. New York, NY. 7.5.2.

27. Eustis, S.L., Boorman, G.A., and Hayashi, Y. 1990. Exocrine pancreas. In Pathology of the Fischer rat. G.A. Boorman, S.L. Eustis, M.R. Elwell, C.A Montgomery, and W.F. MacKenzie, editors. Academic Press. San Diego, CA. $95-108$.

28. Ornitz, D.M., Hammer, R.E., Messing, A., Palmiter, R.D., and Brinster, R.L. 1987. Pancreatic neoplasia induced by SV40-T antigen expression in acinar cells of transgenic mice. Science. 238:188-193.

29. Quaife, C.J., Pinkert, C.A., Ornitz, D.M., Palmiter, R.D., and Brinster, R.L. 1987. Pancreatic neoplasia induced by ras expression in acinar cells of transgenic mice. Cell. 48:1023-1034.

30. Böttinger, E.P., et al. 1997. Expression of a dominant-negative mutant TGF- $\beta$ type II receptor in transgenic mice reveals essential roles for TGF$\beta$ in regulation of growth and differentiation in the exocrine pancreas. EMBO J. 16:2621-2633.

31. Achler, C., Filmer, D., Merte, C., and Drenckhahn, D. 1989. Role of microtubules in polarized delivery of apical membrane proteins to the brush border of the intestinal epithelium. J. Cell Biol. 109:179-189.

32. Parczyk, K., Haase, W., and Kondor-Koch, C. 1989. Microtubules are involved in the secretion of proteins at the apical cell surface of the polarized epithelial cell, Madin-Darby canine kidney. J. Biol. Chem. 264:16837-16846.

33. Drenckhahn, D., and Mannherz, H.G. 1983. Distribution of actin and the actin-associated proteins myosin, tropomyosin, alpha-actin, vinculin, and villin in rat and bovine exocrine glands. Eur. J. Cell Biol. 30:167-176.

34. Jungermann, J., et al. 1995. Disassembly of rat pancreatic acinar cell cytoskeleton during supramaximal secretagogue stimulation. Am.J. Physiol. 268:G328-G338.

35. Paramio, J.M., et al. 1999. Modulation of cell proliferation by cytokeratins K10 and K16. Mol. Cell. Biol. 19:3086-3094.

36. Klöppel, G. 1993. Pathology of nonendocrine pancreatic tumors. In The pancreas. Biology, pathobiology, and disease. V.L.W. Go, et al., editors. Raven Pres. New York, NY. 871-897.

37. Oertel, J.E. 1989. The pancreas: non-neoplastic alterations. Am. J. Surg. Pathol. 13:50-65.

38. Bockman, D.E., Boydston W.R., and Anderson M.C. 1982. Origin of ductular complexes in human chronic pancreatitis. Am. J. Surg. 144:243-249.

39. Bockman, D.E. 1981. Cells of origin of pancreatic cancer: experimental animal tumors related to human pancreas. Cancer. 47(Suppl. 6):1528-1534.

40. Schüssler, M.M., Skoudy, A., Ramaekers, F., and Real, F.X. 1992. Intermediate filaments as differentiation markers of normal pancreas and pancreas cancer. Am. J. Pathol. 140:559-568.

41. Vilá, M.R., Lloreta, J., and Real, F.X. 1994. Normal human pancreas cultures display functional ductal characteristics. Lab. Invest. 71:423-431.

42. De Lisle, R.C., and Logsdon, C.D. 1990. Pancreatic acinar cells in culture: expression of acinar and ductal antigens in a growth-related manner. Eur. J. Cell Biol. 51:64-75.

43. Scarpelli, D.G., Rao, M.S., and Reddy, J.K. 1991. Are acinar cells involved in the pathogenesis of ductal adenocarcinoma of the pancreas? Cancer Cells. 3:275-277.

44. Logsdon, C.D. 1995. Pancreatic duct cell cultures: there is more to ducts than salty water. Gastroenterology. 109:1005-1009.

45. Sandgren, E.P., Quaife, C.J., Paulovich, A.C., Palmiter, R.D., and Brinster, R.L. 1991. Pancreatic tumor pathogenesis reflects the causative genetic lesion. Proc. Natl. Acad. Sci. USA. 88:93-97.

46. Sandgren, E.P, Luetteke, N.C., Palmiter, R.D., Brinster, R.L., and Lee, D.C. 1990. Overexpression of TGF-alpha in transgenic mice: induction of epithelial hyperplasia, pancreatic metaplasia and carcinoma of the breast. Cell. 61:1121-1135.

47. Jhappan, C., et al. 1990. TGF- $\alpha$ overexpression in transgenic mice induces liver neoplasia and abnormal development of the mammary gland and pancreas. Cell. 61:1137-1146.

48. Bockman, D.E., and Merlino, G. 1992. Cytological changes in the pancreas of transgenic mice overexpressing transforming growth factor alpha. Gastroenterology. 103:1883-1892.

49. Chu, W.W., Runyan, R.B., Oshima, R.G., and Hendrix, M.J.C. 1993. Expression of complete keratin filaments in mouse L cells augments cell migration and invasion. Proc. Natl. Acad. Sci. USA. 90:4261-4265.

50. Chu, Y.W., Seftor, E.A., Romer, L.H., and Hendrix, M.J.C. 1996. Experimental coexpression of vimentin and keratin intermediate filaments in human melanoma cells augments motility. Am. J. Pathol. 148:63-69.

51. Hendrix, M.J.C., et al. 1992. Coexpression of vimentin and keratins by human melanoma tumor cells: correlation with invasive and metastatic potential. J. Natl. Cancer Inst. 84:165-174.

52. Cheng, C., Kilkenny, A., Roop, D., and Yuspa, S. 1990. The v-ras oncogene inhibits the expression of differentiation markers and facilitates expression of cytokeratins 8 and 18 in mouse keratinocytes. Mol. Carcinog. 3:363-373

53. Díaz-Guerra, M., et al. 1992. Expression of simple epithelial cytokeratins in mouse epidermal keratinocytes harboring Harvey ras gene alterations. Cancer Res. 52:680-687.

54. Caulín, C., Bauluz, C., Gandarillas, A., Cano, A., and Quintanilla, M. 1993. Changes in keratin expression during malignant progression of transformed mouse epidermal keratinocytes. Exp. Cell Res. 204:11-21. 